\title{
Charged-particle spectroscopy in organic semiconducting single crystals
}

A. Ciavatti, P. J. Sellin, L. Basiricò, A. Fraleoni-Morgera, and B. Fraboni

Citation: Appl. Phys. Lett. 108, 153301 (2016); doi: 10.1063/1.4945597

View online: http://dx.doi.org/10.1063/1.4945597

View Table of Contents: http://aip.scitation.org/toc/apl/108/15

Published by the American Institute of Physics

Applied Physics Reviews 


\title{
Charged-particle spectroscopy in organic semiconducting single crystals
}

\author{
A. Ciavatti, ${ }^{1}$ P. J. Sellin, ${ }^{2}$ L. Basiricò, ${ }^{1}$ A. Fraleoni-Morgera, ${ }^{3,4,5}$ and B. Fraboni ${ }^{1}$ \\ ${ }^{1}$ Department of Physics and Astronomy, University of Bologna, Viale Berti Pichat 6/2, 40127 Bologna, Italy \\ ${ }^{2}$ Department of Physics, University of Surrey, Guildford, Surrey GU2 7XH, United Kingdom \\ ${ }^{3}$ ELETTRA-Sincrotrone Trieste, Strada Statale 14, Km 163.5, Basovizza, Trieste, Italy \\ ${ }^{4}$ Department of Engineering and Architecture, University of Trieste, V. Valerio 10, 34100 Trieste, Italy \\ ${ }^{5}$ CNR-Nano S3 Institute, Via Campi 213/A, 41125 Modena, Italy
}

(Received 10 December 2015; accepted 25 March 2016; published online 11 April 2016)

\begin{abstract}
The use of organic materials as radiation detectors has grown, due to the easy processability in liquid phase at room temperature and the possibility to cover large areas by means of low cost deposition techniques. Direct charged-particle detectors based on solution-grown Organic Semiconducting Single Crystals (OSSCs) are shown to be capable to detect charged particles in pulse mode, with very good peak discrimination. The direct charged-particle detection in OSSCs has been assessed both in the planar and in the vertical axes, and a digital pulse processing algorithm has been used to perform pulse height spectroscopy and to study the charge collection efficiency as a function of the applied bias voltage. Taking advantage of the charge spectroscopy and the good peak discrimination of pulse height spectra, an Hecht-like behavior of OSSCs radiation detectors is demonstrated. It has been possible to estimate the mobility-lifetime value in organic materials, a fundamental parameter for the characterization of radiation detectors, whose results are equal to $\mu \tau_{\text {coplanar }}=(5.5 \pm 0.6) \times 10^{-6} \mathrm{~cm}^{2} / \mathrm{V}$ and $\mu \tau_{\text {sandwich }}=(1.9 \pm 0.2) \times 10^{-6} \mathrm{~cm}^{2} / \mathrm{V}$, values comparable to those of polycrystalline inorganic detectors. Moreover, alpha particles Time-of-Flight experiments have been carried out to estimate the drift mobility value. The results reported here indicate how charged-particle detectors based on OSSCs possess a great potential as low-cost, large area, solid-state direct detectors operating at room temperature. More interestingly, the good detection efficiency and peak discrimination observed for chargedparticle detection in organic materials (hydrogen-rich molecules) are encouraging for their further exploitation in the detection of thermal and high-energy neutrons. (C) 2016 AIP Publishing LLC.

[http://dx.doi.org/10.1063/1.4945597]
\end{abstract}

In recent years, the interest in the potential use of organic materials as radiation detectors has grown, due to their unique properties. In particular, the easy processability in liquid phase at room temperature, and the possibility to cover large areas by means of low cost deposition techniques (e.g., inkjet printing) onto flexible substrates, could overcome some disadvantages of typical inorganic semiconductor detectors, such as silicon and diamond, commonly used for high energy radiation detection and many others ( $\mathrm{SiC}, \mathrm{CdTe}, \mathrm{CZT}, \mathrm{TlBr}$ ), which require high temperature and slow rate growth procedures. Moreover, they cannot cover large areas (i.e., CVD diamond detectors are limited to $1 \mathrm{~cm}^{2}$ in its polycrystalline ${ }^{1}$ form and to few $\mathrm{mm}^{2}$ in single crystal form), unless a large number of inorganic detectors are assembled together ${ }^{2}$ (i.e., in high-energy physics detection application ${ }^{3}$ ), which significantly increases the manufacturing costs.

Organic materials have been investigated as radiation detectors mainly as organic photodiodes coupled to a scintillator $^{4}$ or as X-ray dosimeters for medical application. ${ }^{5-7}$ To date, very little work has been reported concerning the use of organic materials as solid-state radiation detectors operating in "pulse mode." In one reported study of alpha particle detection in pulse mode using polyacetylene foils, ${ }^{8}$ the devices exhibited a very unstable behavior in time when exposed to oxygen and water, and a charge drift velocity of $40 \mathrm{~cm} / \mathrm{s}$ was measured under dry argon atmosphere. More recently, polyaniline has also been studied as an alpha- and betaparticle detector. This material showed greater stability in air than polyacetylene, but it exhibited limited detection efficiency and no spectroscopy grade performance. ${ }^{9}$

Among organic materials, Organic Semiconducting Single Crystals (OSSCs) have been recently proven to be rather robust to environmental conditions (air, light, humidity), ${ }^{10}$ and to have unique transport properties, due to their high chemical purity and lack of grain boundaries typical of polymeric films. In particular, they exhibit charge transport anisotropy, ${ }^{11}$ high charge carrier mobility if compared to organic thin films (the highest reported among organic materials, up to $20 \mathrm{~cm}^{2} / \mathrm{V} \mathrm{s}$ for rubrene single crystal-based transistors ${ }^{12}$ ), and long exciton diffusion lengths, up to $8 \mu \mathrm{m},{ }^{13}$ a very high value compared to the $10 \mathrm{~nm}$ reported for organic thin-film photovoltaic devices. ${ }^{14}$

In the framework of ionizing radiation detectors, we have recently demonstrated how solution grown OSSCs can be employed as solid-state direct X-ray detectors. These detectors are able to operate in ambient atmosphere, environmental light, and room temperature, providing a stable, linear photocurrent response with increasing dose rate and very good radiation hardness properties. ${ }^{10,15}$ Considering these promising results in X-ray sensing and the above described unique properties of OSSCs, their further exploitation in charged-particle detection (i.e., alpha and beta radiation) appears to be extremely interesting. In addition, OSSC detectors have the potential to be used as fast neutron solid-state detectors, due to the high number of proton recoil events within hydrogen-rich organic molecules. 
In this paper, we report on the experimental demonstration of pulse-mode charged-particle detection by a solutiongrown OSSC, namely, 4-hydroxycyanobenzene (4HCB), characterized using pulse-height analysis and rise time analysis of pulses generated by alpha particles in air and at room temperature. Due to the relatively long carrier drift time in these organic devices, a digital pulse processing algorithm has been used to perform pulse height spectroscopy and to study the charge collection efficiency (CCE) as a function of applied bias voltage. Drift mobility measurements of the charge carriers have also been carried out using alpha particle Time-Of-Flight (TOF) measurements.

The detectors were fabricated with $200 \mu \mathrm{m}$ thick $4 \mathrm{HCB}$ ultrapure single crystals (accurate FT-IR analysis showed no trace of foreign compounds in the crystals ${ }^{16}$ ) with $2-4 \mathrm{~mm}^{2}$ of surface area. These crystals were grown from $3 \mathrm{mg} / \mathrm{ml}$ solutions in ethylic ether at $6{ }^{\circ} \mathrm{C}$, using $4 \mathrm{HCB}$ crystals as starting material pre-purified by multiple, subsequent crystallizations (up to 4), again in ethylic ether, as previously described. ${ }^{17,18}$ The 4HCB molecule has been chosen for its robustness to ambient conditions, and for the size of its platelet-shaped single crystals that can be easily tuned to obtain large (several $\mathrm{mm}^{2}$ ) and thick (up to $1 \mathrm{~mm}$ ) samples (Figure 1(a)). Moreover, we decided to test self standing $\mathrm{mm}$-sized 4HCB crystals for alpha detection, because an alternative process which is able to deliver large area crystals, i.e., inkjet printing of solutions from which the crystals can grow, is still under optimization. Importantly, 4HCB single crystals already demonstrated interesting photocurrent detection performances ${ }^{10}$ and optimized charge collection efficiency ${ }^{19}$ under X-ray irradiation.

We exploited the already studied anisotropy of $4 \mathrm{HCB}$ single crystals (electrical transport ${ }^{17}$ and $\mathrm{X}$-ray response ${ }^{15}$ ) considering both a coplanar and a sandwich electrodes configuration, addressing the planar and the vertical crystallographic axes, respectively. Electrical contacts were fabricated in the coplanar structure, with pads $400 \mu \mathrm{m}$ away from each other, and in the sandwich configuration with $100 \mathrm{~nm}$ thick sputtered gold electrodes on both sides of crystals, forming a metal-semiconductor-metal (MSM) device structure. Figures 1(b) and 1(c) show the electrical characterization of typical coplanar and sandwich electrode geometry devices (sketch in inset), as measured with a Keithley Picoammeter 487. The electrical injection is ohmic in both configurations and the current density is low, as it is typical for large energy gap semiconductors. The resistivity values are $\rho_{\text {coplanar }}=(8.8 \pm 0.7) \times 10^{10} \Omega \mathrm{cm}$ and $\rho_{\text {sandwich }}=$ $(6.27 \pm 0.05) \times 10^{9} \Omega \mathrm{cm}$, respectively.

Irradiation was performed in air at room temperature using a ${ }^{241} \mathrm{Am}$ alpha source, with the particles impinging on the top surface. In the sandwich configuration, the bias voltage was applied to the electrode on the top surface, with the bottom electrode connected to the ground. Bias voltages in the range of $100-800 \mathrm{~V}$ were used in coplanar configuration, and up to $500 \mathrm{~V}$ in sandwich configuration: at higher voltages, the current flowing in the crystals is noisy and unstable, producing large spikes at the preamplifier input that masked the alpha signal. The uncollimated $5.49 \mathrm{MeV}{ }^{241} \mathrm{Am}$ source, with an activity of $185 \mathrm{kBq}$, was positioned inside a metal chamber at a distance of $5 \mathrm{~mm}$ from the device. At this distance, taking into account the mean energy loss in air, alpha particles with an average energy of $5.02 \mathrm{MeV}$ impinge on the organic crystal surface which are stopped in approximately $31 \mu \mathrm{m}$ inside the crystal. The mean energy loss in air and the alpha particles range were calculated by the employment of the software package SRIM for the simulation of stopping and range of ions in matter. ${ }^{20}$

The OSSCs detectors were connected to an ultra low noise charge-sensitive preamplifier ( $\mathrm{eV}$ model 550 ), with a charge sensitivity of $3.6 \mathrm{mV} / \mathrm{fC}$. The effective rise-time of the preamplifier circuit was $160 \mathrm{~ns}$, much shorter than the signal rise time detected, and the decay time of the preamplifier was longer than $1 \mathrm{~ms}$. Such long decay time is essential for avoiding incorrect estimation of slow organic signals. Bias voltage was applied via the preamplifier by means of an Ortec 710 power supply. The preamplifier output was directly connected to a four-channel digital oscilloscope (Tektronix TBS3034B, $300 \mathrm{MHz}$ bandwidth), triggered on each pulse, and the pulse waveforms ( $200 \mu$ s time window) were captured on an

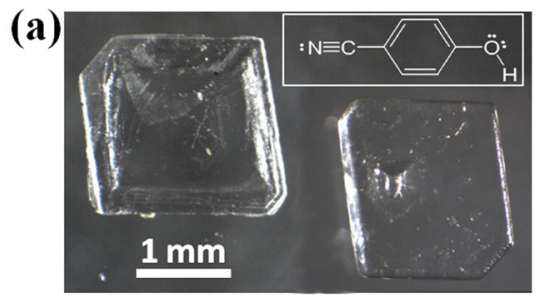

(b) coplanar

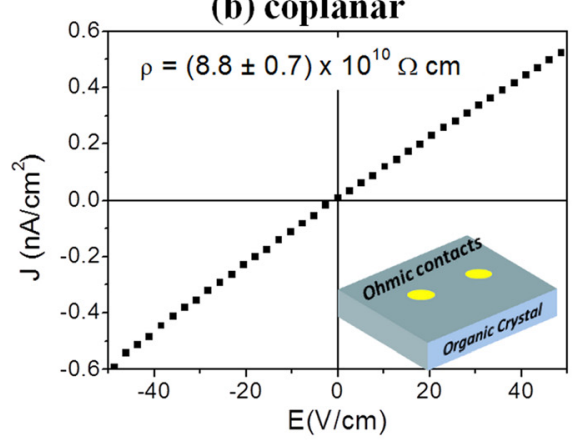

(c) sandwich

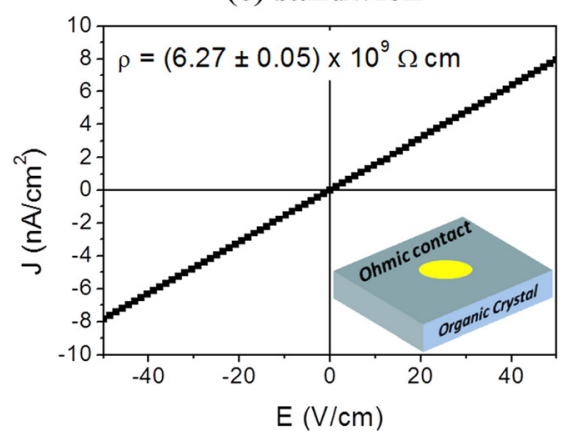

FIG. 1. (a) Optical image of mm-sized 4HCB single crystals and the corresponding molecular formula. Currentvoltage characteristic of 4HCB single crystals $200 \mu \mathrm{m}$ thick with coplanar (b) and sandwich (c) electrodes geometry. 


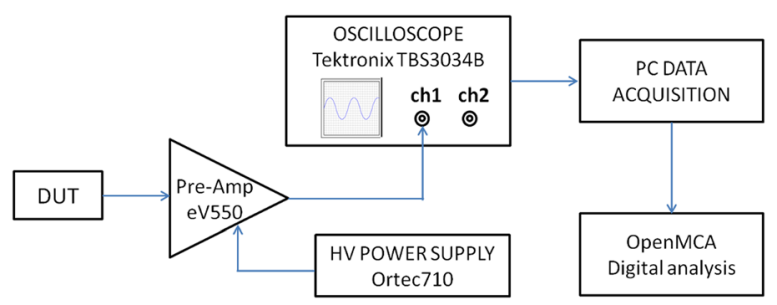

FIG. 2. Block diagram of the experimental setup. The organic crystal (DUT) is directly connected to charge-sensitive preamplifier eV550. The radiation pulses are digitalized by the oscilloscope and processed by OpenMCA software.

event-by-event basis and digitally stored for off-line analysis (Figure 2). The analysis software, written in LabView, provided a digital pulse shaper with long shaping times, in the order of tens of microseconds (much longer than those typically required for inorganic detectors). The analysis algorithm used a digital CR-RC shaper to measure the pulse amplitude, and the rise-time of the preamplifier pulse measured from $10 \%$ to $90 \%$ of the pulse amplitude. The algorithm is described in more detail in Ref. 21. A shaping time of $50 \mu \mathrm{s}$ for the CR-RC shaper was used in this work, with the pulse height binned across 512 channels.

Figure 3(a) shows an $\alpha$-pulse in 4HCB OSSCs devices for ${ }^{241} \mathrm{Am}$ alpha particles $\left(\mathrm{A}=1.85 \times 10^{5} \mathrm{~Bq}\right)$, as acquired at the preamplifier output. The figure indicates the $10 \%-90 \%$ rise-time of the preamplifier pulse in the order of tens of microseconds, corresponding to the charge drift time, which is much longer than the nanosecond drift times typical of inorganic semiconductor detectors. Indeed, we have to keep in mind the very slow drift times typical of organic materials that affect the pulsed measurements, ${ }^{22,23}$ in order to avoid large dead time and high charge losses in the acquired pulse height spectra. We used a digital CR-RC shaper and multichannel analyzer, which allows selection of a wide range of shaping times of the signal, in order to overcome the limit of short shaping time from analog shaping amplifiers. We tested several shaping times between $10 \mu \mathrm{s}$ and $150 \mu \mathrm{s}$, and we set a shaping time of $50 \mu \mathrm{s}$ as the best trade off between charge losses, electrical noise (relevant at short shaping time), energy resolution and pile-up effects (relevant at longer shaping time). The appropriate shaping time reduces the charge loss during the shaping of the signal, and therefore, by employing digital analysis, the detector shows broad but well-resolved full-energy peaks, e.g., in the spectrum obtained from about 10000 triggered pulses (black line in Figure 3(b)) for a $4 \mathrm{HCB}$ single crystal detector biased at $\mathrm{V}=300 \mathrm{~V}$. The alpha peak is well resolved compared to the background noise (red line in Figure 3(b)) acquired from the same detector removing the ${ }^{241} \mathrm{Am}$ source; in particular, the trigger level was lowered in order to allow the acquisition of some signal from the background. The experiment was then repeated in the same conditions of Figure 3(b) but with a paper foil interposed between the alpha source and the detector. The paper was thick enough $(\approx 100 \mu \mathrm{m})$ to stop all the alpha particles but not the gamma rays coming out from the same ${ }^{241} \mathrm{Am}$ source. The data obtained from the sample with shielded source are identical to the background contribution, confirming that the peak observed with the unshielded alpha source is due to alpha particles.
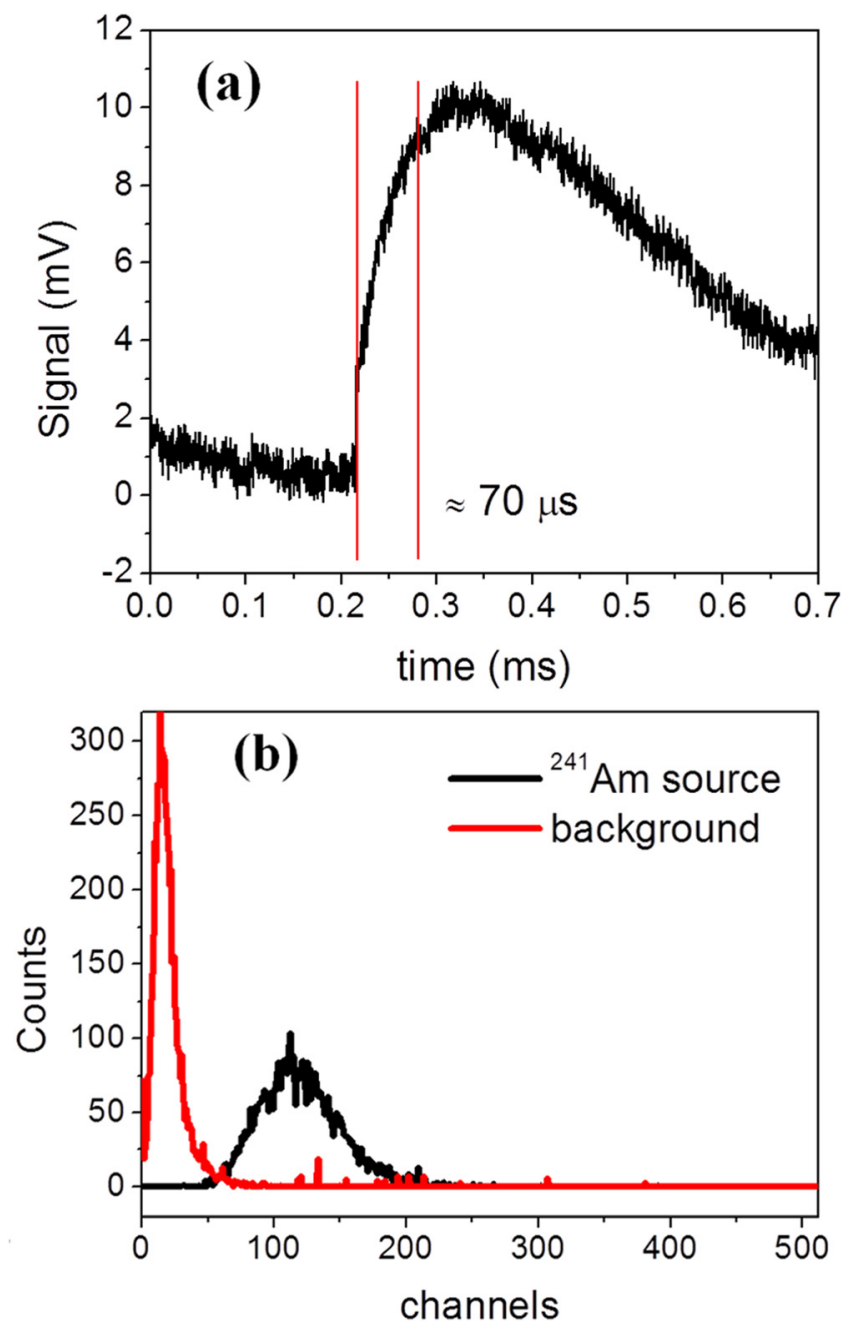

FIG. 3. (a) Typical ${ }^{241} \mathrm{Am}$ pulse in $4 \mathrm{HCB}$ single crystal at $\mathrm{V}=500 \mathrm{~V}$. Vertical red lines indicate the rise time of the pulse (10\%-90\% of the full amplitude). (b) Digital processed pulse height spectrum of ${ }^{241} \mathrm{Am}$ peak (black) compared to the background noise (red) for $\mathrm{V}=300 \mathrm{~V}$ and $50 \mu$ s of shaping time.

In Figures 4(a) and 4(b), the digital pulse height spectra are reported as a function of applied bias voltages for the coplanar and sandwich structures, respectively. The detectors show full-energy peaks over the full range of bias voltages and clearly indicate a reduced charge loss. Moreover, due to better charge collection efficiency, the position of the peak centroid shifts toward higher channels with increasing bias voltage. The digital pulse height spectra observed for OSSCs in Figure 4 represent a dramatic improvement in spectroscopy resolution compared to earlier reports on organic polymeric detectors based on gold wire anodes. ${ }^{9}$ It is also important to stress that the OSSCs-based detectors here reported work at much lower bias values than organic polymeric film (bias $>1000 \mathrm{~V})$. These devices are also stable in air and ambient light, and thus do not require controlled atmosphere to avoid detector degradation. ${ }^{8}$ These good results have to be ascribed to the use of organic single crystals that have better charge transport properties, less impurities than polymers and thin films, and to the employment of digital shaping that strongly reduces the charge loss. The position shift of the Gaussian peak centroid extracted from the pulse height spectra for coplanar and sandwich configuration is plotted in Figures 5(a) and 5(b), respectively, as a function of bias voltage. 

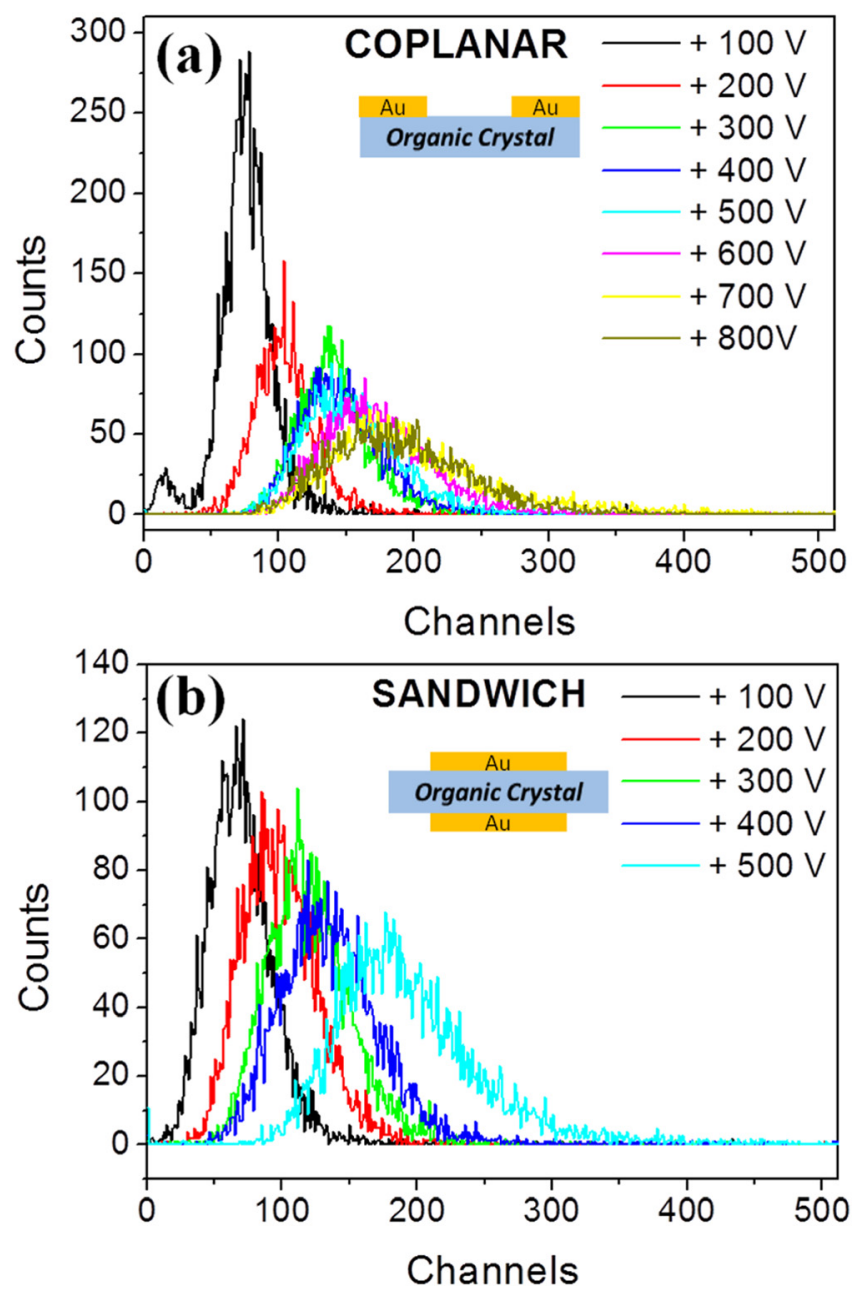

FIG. 4. Pulse height spectra obtained with digital pulse shape analysis at shaping time of $50 \mu \mathrm{s}$. (a) Coplanar and (b) sandwich electrodes configuration.

The peak centroid channel value is proportional to the charge collection efficiency $\eta$ (i.e., the fraction of energy detected over the total theoretical energy release in the material). The peak centroid shift at higher channels increasing the bias voltage indicates an improvement in charge collection efficiency. In addition, the centroid peak tends to saturate at high voltages, with a well known behavior, observed also in inorganic detectors, which follow the Hecht plot function $^{24}$

$$
\eta=\frac{\mu \tau \cdot V}{d^{2}}\left[1-\exp \left(-\frac{d^{2}}{\mu \tau \cdot V}\right)\right]
$$

where $V$ is the applied voltage, $d$ is the electrodes distance, and $\mu \tau$ is the mobility-lifetime product of the detector material. The fitting of experimental data is reported in the red lines in Figures 6(a) and 6(b), and the $\mu \tau$ value extracted from the fitted curve results equals to $\mu \tau_{\text {coplanar }}=(5.5 \pm 0.6) \times 10^{-6}$ $\mathrm{cm}^{2} / \mathrm{V}$ and $\mu \tau_{\text {sandwich }}=(1.9 \pm 0.2) \times 10^{-6} \mathrm{~cm}^{2} / \mathrm{V}$, values comparable to those of polycrystalline CdTe and CZT detectors. $^{25,26}$ In this analysis, we have applied the regular Hecht equation to the pulse height data, which assumes a uniform electric field distribution through the active volume of the device. The application of this technique to organic detectors is of great interest for the future, and more work is required to
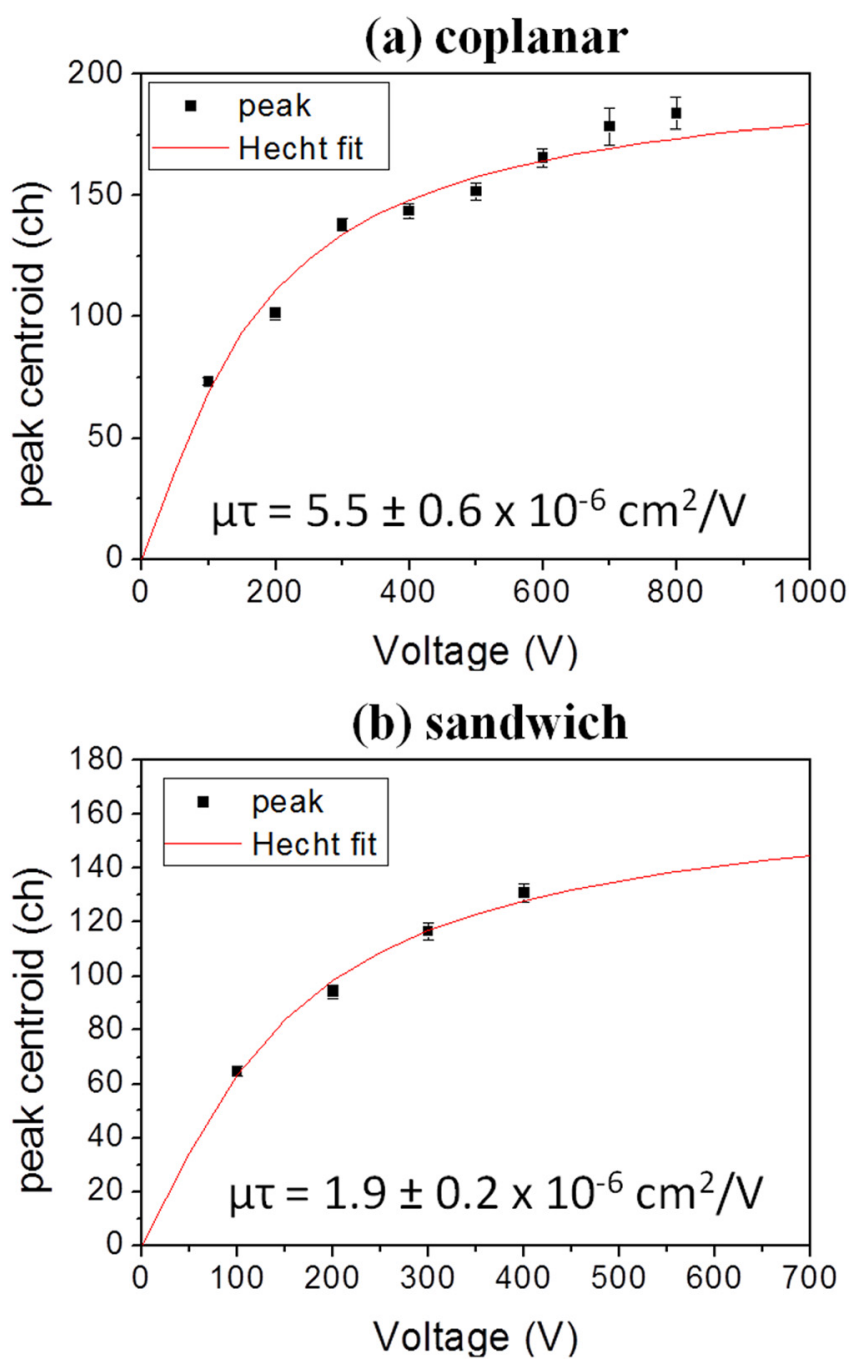

FIG. 5. Gaussian peak centroid position vs. applied voltage plot (black dot) for (a) coplanar and (b) sandwich configurations (anode irradiation). The red lines show the Hecht equation fit (Eq. (1)) to the experimental data. Mobility-lifetime results are also reported.

establish the exact nature of the charge transport mechanism in these devices. Nevertheless, organic single crystal detectors have Hecht-like behavior and it has been possible to estimate the mobility-lifetime value, a fundamental parameter for the characterization of radiation detectors, in organic materials.

Taking further advantage of the low penetration depth of alpha particles in materials, we can perform time-of-flight analysis on sandwich geometry devices, as described in Ref. 27 for polycrystalline diamond. It should be stressed that all the spectra reported so far have been obtained for positive applied biases: this does not make any difference in coplanar geometry because the contacts are ohmic and the alpha particles are stopped close to the crystal surface, thus both electrons and holes are collected at the same time by the two electrodes. However, in the sandwich configuration with the top electrode biased as the anode, the signal is due only to holes drifting from near the top surface to the bottom electrode. Therefore, the pulse rise-time gives a measure of the drift time of holes in the device. For each pulse, the $20 \%-80 \%$ pulse rise time has been measured and the histogram was plotted, as shown in Figure 3(a). The histogram of the pulse rise-time shows a Gaussian distribution (Figure 

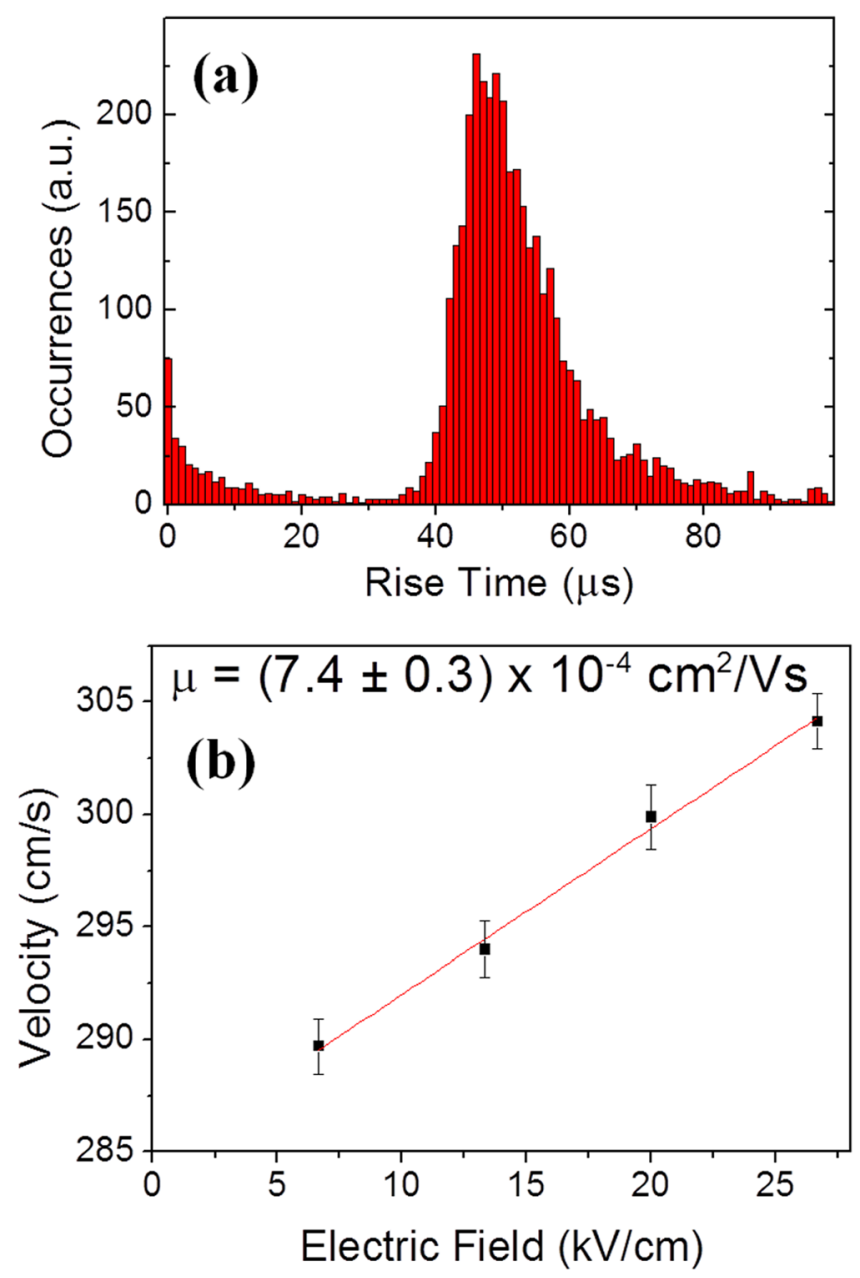

FIG. 6. Time-of-flight experiment with alpha particles probe in 4HCB single crystals in sandwich configuration. (a) Pulse rise time histogram of 4HCB single crystal biased at $200 \mathrm{~V}$ and (b) mobility value extrapolation from drift velocity vs. electric field linear plot.

$6(\mathrm{a}))$, where the mean rise-time decreases from $51.8 \mu \mathrm{s}$ at $+100 \mathrm{~V}$ to $49.3 \mu \mathrm{s}$ at $+400 \mathrm{~V}$. Figure $6(\mathrm{~b})$ reports the corresponding hole drift velocity as a function of the electric field. The drift velocity value, up to $305 \mathrm{~cm} / \mathrm{s}$, is much higher than that reported for polymers with the same characterization techniques $(40 \mathrm{~cm} / \mathrm{s}),{ }^{8}$ underlining the better charge transport in organic single crystals. The hole mobility value, extracted from the linear fit of the velocity vs. electric field plot, is equal to $(7.4 \pm 0.3) \times 10^{-4} \mathrm{~cm}^{2} / \mathrm{V} \mathrm{s}$, which is in very good agreement with the reported mobility values along the same axis measured with space-charge-limited current method, for the best performing 4HCB single crystals. ${ }^{17}$

In conclusion, the assessments of alpha particles detection capability of organic semiconducting single crystals have been achieved using long shaping time, with very good charge collection and peak discrimination, opening the way to the employment of organic materials in charge counting spectroscopy of radiation sources; more interestingly, the detection efficiency observed for charged-particle detection in organic materials (hydrogen-rich molecules) is encouraging for their further exploitation in the direct detection of thermal and high energy neutrons. Moreover, the reported results open the possibility of employing alpha particles as a tool for the organic materials investigation, using charge collection efficiency measurements to extract $\mu \tau$ value and time-of-flight measurements to measure drift velocity and mobility. Further investigations are being carried out in order to determine the electron-hole pair creation energy in organic materials, and thus to quantitatively estimate the $\mathrm{CCE}$ of OSSC-based radiation detectors.

The authors acknowledge the financial support from the European Community under the FP7-ICT Project "i-FLEXIS" (2013-2016). Grant Agreement No. 611070.

${ }^{1}$ A. Gorišek, V. Cindro, I. Dolenc, H. Frais-Kölbl, E. Griesmayer, H. Kagan, S. Korpar, G. Kramberger, I. Mandić, M. Meyer et al., Nucl. Instrum. Methods Phys. Res. Sect. A 572, 67 (2007).

${ }^{2}$ M. Girolami, A. Bellucci, P. Calvani, C. Cazzaniga, M. Rebai, D. Rigamonti, M. Tardocchi, M. Pillon, and D. M. Trucchi, Phys. Status Solidi A 212, 2424 (2015).

${ }^{3}$ The ATLAS Collaboration, G. Aad, E. Abat, J. Abdallah, A. A. Abdelalim, A. Abdesselam, O. Abdinov, B. A. Abi, M. Abolins, H. Abramowicz, E. Acerbi et al., J. Instrum. 3, S08003 (2008).

${ }^{4}$ E. Takada, K. Fujii, H. Imai, H. Okada, Y. Namito, and T. Nakamura, J. Nucl. Sci. Technol. 52, 104 (2015).

${ }^{5}$ F. A. Boroumand, M. Zhu, A. B. Dalton, J. L. Keddie, P. J. Sellin, and J. J. Gutierrez, Appl. Phys. Lett. 91, 033509 (2007).

${ }^{6}$ C. A. Mills, H. Al-Otaibi, A. Intaniwet, M. Shkunov, S. Pani, J. L. Keddie, and P. J. Sellin, J. Phys. Appl. Phys. 46, 275102 (2013).

${ }^{7}$ D. Natali and M. Sampietro, Nucl. Instrum. Methods Phys. Res. Sect. A 512, 419 (2003).

${ }^{8}$ P. Beckerle and H. Ströbele, Nucl. Instrum. Methods Phys. Res. Sect. A 449, 302 (2000).

${ }^{9}$ T. Suzuki, H. Miyata, M. Katsumata, S. Nakano, K. Matsuda, and M. Tamura, Nucl. Instrum. Methods Phys. Res. Sect. A 763, 304 (2014).

${ }^{10}$ B. Fraboni, A. Ciavatti, F. Merlo, L. Pasquini, A. Cavallini, A. Quaranta, A. Bonfiglio, and A. Fraleoni-Morgera, Adv. Mater. 24, 2289 (2012).

${ }^{11}$ D. Braga, N. Battaglini, A. Yassar, and G. Horowitz, Phys. Rev. B 77, 115205 (2008).

${ }^{12}$ V. Podzorov, E. Menard, A. Borissov, V. Kiryukhin, J. Rogers, and M. Gershenson, Phys. Rev. Lett. 93, 086602 (2004).

${ }^{13}$ H. Najafov, B. Lee, Q. Zhou, L. C. Feldman, and V. Podzorov, Nat. Mater. 9, 938 (2010).

${ }^{14}$ K.-J. Baeg, M. Binda, D. Natali, M. Caironi, and Y.-Y. Noh, Adv. Mater. 25, 4267 (2013).

${ }^{15}$ B. Fraboni, A. Ciavatti, L. Basiricò, and A. Fraleoni-Morgera, Faraday Discuss. 174, 219 (2014).

${ }^{16}$ E. Capria, L. Benevoli, A. Perucchi, B. Fraboni, M. Tessarolo, S. Lupi, and A. Fraleoni-Morgera, J. Phys. Chem. A 117, 6781 (2013).

${ }^{17}$ B. Fraboni, C. Femoni, I. Mencarelli, L. Setti, R. Di Pietro, A. Cavallini, and A. Fraleoni-Morgera, Adv. Mater. 21, 1835 (2009).

${ }^{18}$ A. Fraleoni-Morgera, L. Benevoli, and B. Fraboni, J. Cryst. Growth 312, 3466 (2010).

${ }^{19}$ A. Ciavatti, E. Capria, A. Fraleoni-Morgera, G. Tromba, D. Dreossi, P. J. Sellin, P. Cosseddu, A. Bonfiglio, and B. Fraboni, Adv. Mater. 27, 7213 (2015).

${ }^{20}$ J. Ziegler, SRIM 2013, see http://www.srim.org.

${ }^{21}$ S. K. Chaudhuri, A. Lohstroh, M. Nakhostin, and P. J. Sellin, J. Instrum. 7, T04002 (2012).

${ }^{22}$ P. Stallinga, H. L. Gomes, H. Rost, A. B. Holmes, M. G. Harrison, and R. H. Friend, Synth. Met. 111-112, 535 (2000).

${ }^{23}$ D. V. Lang, X. Chi, T. Siegrist, A. M. Sergent, and A. P. Ramirez, Phys. Rev. Lett. 93, 076601 (2004).

${ }^{24}$ G. F. Knoll, Radiation Detection and Measurement, 4th ed. (Wiley, 2011).

${ }^{25}$ K. Yang, B. Cha, D. Heo, and S. Jeon, Phys. Status Solidi C 11, 1341 (2014).

${ }^{26}$ K. H. Kim, Y. H. Na, Y. J. Park, T. R. Jung, S. U. Kim, and J. K. Hong, IEEE Trans. Nucl. Sci. 51, 3094 (2004).

${ }^{27}$ S. Wang, P. Sellin, and A. Lohstroh, Carbon 43, 3167 (2005). 